\title{
Regioselective Reductive Coupling of Alkynes and Aldehydes Leading to Allylic Alcohols
}

\author{
Kazuhiko Takai,* Shuji Sakamoto, and Takahiko Isshiki \\ Department of Applied Chemistry, Faculty of Engineering,
} Okayama University, Tsushima, Okayama 700-8530, Japan

Experimental Conditions: Unless otherwise noted, materials were obtained from commercial suppliers and were used without further purification. Dry, oxygen-free dimethylformamide (DMF) was purchased from Kanto Chemicals, Co. Column chromatography was done with silica gel (200 mesh). Distillation of small amounts of products was performed with a Büchi Kugelrohr, and boiling points are indicated by an air bath temperature without correction. FT-IR spectra were obtained on a Nicolet Protégé 460 spectrometer. ${ }^{1} \mathrm{H}$ and ${ }^{13} \mathrm{C}$ NMR spectra were recorded on a JEOL JNM-LA400 instrument. Chemical shifts are expressed in ppm downfield from internal tetramethylsilane using the $\delta$ scale. Elemental analyses were performed by the staff at the Elemental Analyses Center of Kyoto University.

General Procedure: To a mixture of $\mathrm{CrCl}_{2}(0.61 \mathrm{~g}, 5.0 \mathrm{mmol})$ and a catalytic amount of $\mathrm{NiCl}_{2}(32 \mathrm{mg}, 0.25 \mathrm{mmol})$ and triphenylphosphine $(0.13 \mathrm{~g}, 0.50 \mathrm{mmol})$ in DMF $(6 \mathrm{~mL})$ was added a solution of an aldehyde $(1.0 \mathrm{mmol})$ in DMF $(4 \mathrm{~mL})$ at $25{ }^{\circ} \mathrm{C}$ and the mixture was stirred for $10 \mathrm{~min}$. A solution of a terminal alkyne $(2.5 \mathrm{mmol})$ and water (1 M of a DMF solution, $4 \mathrm{~mL})$ in DMF $(6$ $\mathrm{mL}$ ) was added at $25^{\circ} \mathrm{C}$ to the mixture over period of $4 \mathrm{~h}$. After stirring at $25^{\circ} \mathrm{C}$ for additional $4 \mathrm{~h}$, the reaction mixture was poured into brine $(30 \mathrm{~mL})$. The mixture was extracted with ether $(3 \times 20$ $\mathrm{mL}$ ), and organic extracts were dried over anhydrous magnesium sulfate and concentrated. Purification by column chromatography on silica gel gave the desired coupling product. 


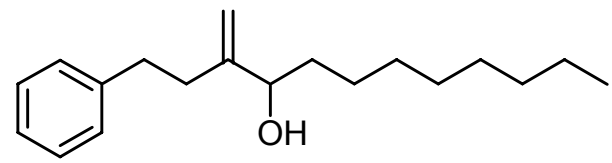

2-(2-Phenylethyl)-1-undecen-3-ol (4): $\quad$ Bp $145{ }^{\circ} \mathrm{C}$ (bath temp, 0.46 Torr); IR (neat): 3355, 3085, 3027, 2927, 2855, 1646, 1604, 1496, 1455, 1076, 1031, 901, 746, $698 \mathrm{~cm}^{-1}$; ${ }^{1} \mathrm{HNMR}$ $\left(\mathrm{CDCl}_{3}\right): \quad \delta 0.88(\mathrm{t}, J=6.9 \mathrm{~Hz}, 3 \mathrm{H}), 1.22-1.33(\mathrm{~m}, 12 \mathrm{H}), 1.38-1.43(\mathrm{~m}, 1 \mathrm{H}), 1.49-1.61(\mathrm{~m}, 2 \mathrm{H})$, $2.29(\mathrm{dt}, J=15.3,8.0 \mathrm{~Hz}, 1 \mathrm{H}), 2.42(\mathrm{dt}, J=15.6,8.0 \mathrm{~Hz}, 1 \mathrm{H}), 2.81(\mathrm{t}, J=8.1 \mathrm{~Hz}, 2 \mathrm{H}), 4.07(\mathrm{~s}, 1 \mathrm{H})$, $4.91(\mathrm{~d}, J=1.2 \mathrm{~Hz}, 1 \mathrm{H}), 5.06(\mathrm{~s}, 1 \mathrm{H}), 7.15-7.34(\mathrm{~m}, 5 \mathrm{H}) ;{ }^{13} \mathrm{CNMR}\left(\mathrm{CDCl}_{3}\right): \delta 14.1,22.7,25.7$, 29.3, 29.5, 29.6, 31.9, 32.9, 34.5, 35.5, 75.7, 109.9, 125.9, 128.3, 128.3, 142.0, 151.5. Anal. Calcd for $\mathrm{C}_{19} \mathrm{H}_{30} \mathrm{O}$ : C, 83.15; H, 11.02. Found: C, 83.31; H, 11.27.

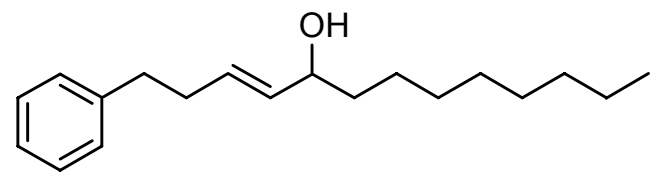

(E)-1-Phenyl-3-tridecen-5-ol (5): ${ }^{1}$ IR (neat): 3337, 3108, 3086, 3027, 2954, 2855, 1604, 1496, 1454, 1377, 1304, 1131, 1030, 969, 746, $698 \mathrm{~cm}^{-1} ;{ }^{1} \mathrm{HNMR}\left(\mathrm{CDCl}_{3}\right): \quad \delta 0.89(\mathrm{t}, J=6.9 \mathrm{~Hz}$, $3 \mathrm{H}), 1.23-1.31(\mathrm{~m}, 12 \mathrm{H}), 1.34-1.37(\mathrm{~m}, 1 \mathrm{H}), 1.41-1.53(\mathrm{~m}, 2 \mathrm{H}), 2.36(\mathrm{dt}, J=7.8,7.1 \mathrm{~Hz}, 2 \mathrm{H}), 2.70$ $(\mathrm{t}, J=7.6 \mathrm{~Hz}, 2 \mathrm{H}), 4.00-4.05(\mathrm{~m}, 1 \mathrm{H}), 5.47$ (ddt, $J=15.3,7.1,1.2 \mathrm{~Hz}, 1 \mathrm{H}), 5.67(\mathrm{dt}, J=15.3,7.1$ $\mathrm{Hz}, 1 \mathrm{H}), 7.17-7.30(\mathrm{~m}, 5 \mathrm{H}) ;{ }^{13} \mathrm{CNMR}\left(\mathrm{CDCl}_{3}\right): \quad \delta 14.1,22.7,25.4,29.3,29.5,29.5,31.9,33.9$, $35.6,37.3,73.1,125.8,128.3,128.4,130.9,133.8,141.7$.

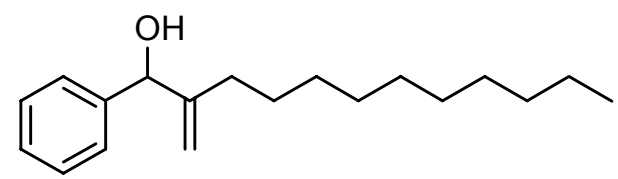

2-Decyl-1-phenyl-2-propen-1-ol: ${ }^{2}$ IR (neat): 3364, 3085, 3063, 3029, 2925, 2853, 1648, 1603, 1493, 1454, 1377, 1190, 1025, 902, 842, $699 \mathrm{~cm}^{-1} ;{ }^{1} \mathrm{HNMR}\left(\mathrm{CDCl}_{3}\right): \quad \delta 0.88(\mathrm{t}, J=6.9 \mathrm{~Hz}$, $3 \mathrm{H}), 1.19-1.31(\mathrm{~m}, 14 \mathrm{H}), 1.34-1.43(\mathrm{~m}, 2 \mathrm{H}), 1.79-1.88(\mathrm{~m}, 2 \mathrm{H}), 1.95(\mathrm{dt}, J=15.6,7.9 \mathrm{~Hz}, 1 \mathrm{H}), 4.98$ $(\mathrm{s}, 1 \mathrm{H}), 5.16(\mathrm{~d}, J=3.3 \mathrm{~Hz}, 1 \mathrm{H}), 5.26(\mathrm{~s} 1 \mathrm{H}), 7.28-7.38(\mathrm{~m}, 5 \mathrm{H}) ;{ }^{13} \mathrm{CNMR}\left(\mathrm{CDCl}_{3}\right): \quad \delta 14.1,22.7$, $27.8,29.3,29.4,29.5,29.55,29.58,31.8,31.9,77.3,109.6,126.7,127.7,128.4,142.2,151.2$.

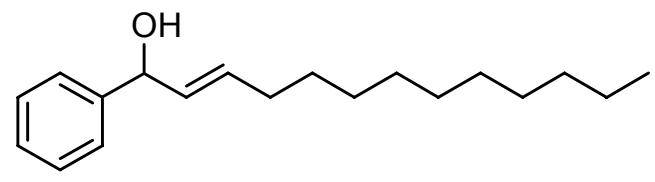

(E)-1-Phenyl-2-tridecen-1-ol: 3 IR (neat): 3347, 3103, 3082, 3060, 2926, 2855, 1599, 1494, 1465, 965, 748, $693 \mathrm{~cm}^{-1} ;{ }^{1} \mathrm{HNMR}\left(\mathrm{CDCl}_{3}\right): \quad \delta 0.86(\mathrm{t}, J=6.8 \mathrm{~Hz}, 3 \mathrm{H}), 1.21-1.28(\mathrm{~m}, 14 \mathrm{H})$, 
1.34-1.41 (m, 2H), $1.90(\mathrm{~s}, 1 \mathrm{H}), 2.03(\mathrm{dt}, J=7.2,7.0 \mathrm{~Hz}, 2 \mathrm{H}), 5.14(\mathrm{~d}, J=6.6 \mathrm{~Hz}, 1 \mathrm{H}), 5.63(\mathrm{dd}, J=$ 15.3, $6.6 \mathrm{~Hz}, 1 \mathrm{H}), 5.74(\mathrm{dt}, J=15.3,6.6 \mathrm{~Hz}, 1 \mathrm{H}), 7.23-7.36(\mathrm{~m}, 5 \mathrm{H}) ;{ }^{13} \mathrm{CNMR}\left(\mathrm{CDCl}_{3}\right): \delta 14.1$, $22.7,29.1,29.2,29.3,29.4,29.6,29.6,31.9,32.2$, 75.2, 126.1, 127.4, 128.4, 132.2, 132.9, 143.4.

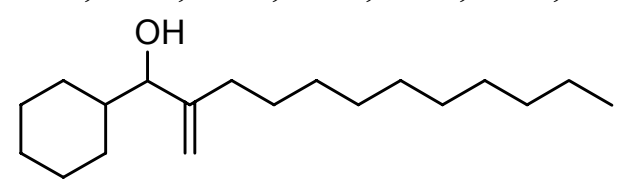

1-Cyclohexyl-2-decyl-2-propen-1-ol: $\quad$ Bp $120^{\circ} \mathrm{C}$ (bath temp, 0.45 Torr); IR (neat): 3398, 2924, 2852, 1645, 1465, 1450, 1378, 1306, 1261, 1082, 1021, 897, 803, 735, $666 \mathrm{~cm}^{-1}$; ${ }^{1} \mathrm{HNMR}$ $\left(\mathrm{CDCl}_{3}\right): \quad \delta 0.88(\mathrm{t}, J=6.8 \mathrm{~Hz}, 3 \mathrm{H}), 0.92-1.05(\mathrm{~m}, 1 \mathrm{H}), 1.11-1.29(\mathrm{~m}, 18 \mathrm{H}), 1.41-1.50(\mathrm{~m}, 5 \mathrm{H})$, $1.63-1.67(\mathrm{~m}, 1 \mathrm{H}), 1.71-1.79(\mathrm{~m}, 2 \mathrm{H}), 1.88-1.96(\mathrm{~m}, 2 \mathrm{H}), 2.06(\mathrm{dt}, J=15.9,7.9 \mathrm{~Hz}, 1 \mathrm{H}), 3.77(\mathrm{~d}, J$

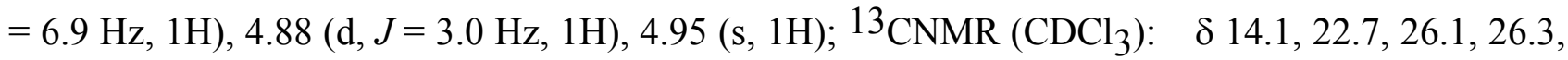
26.5, 28.0, 28.1, 29.4, 29.59, 29.63, 29.63, 29.7, 29.9, 31.2, 31.9, 41.0, 80.7, 110.3, 151.0. Anal. Calcd for $\mathrm{C}_{19} \mathrm{H}_{36} \mathrm{O}$ : C, 81.36; H, 12.94. Found: C, 81.43; H, 13.08.

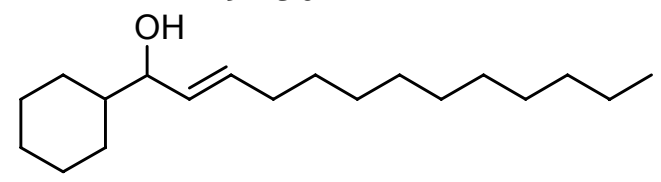

(E)-1-Cyclohexyl-2-tridecen-1-ol: $\quad$ IR (neat): $\quad 3371,2925,2853,1669,1464,1450,1378$, 1306, 1261, 1084, 1004, 969, 892, 721, $666 \mathrm{~cm}^{-1} ;{ }^{1} \mathrm{HNMR}\left(\mathrm{CDCl}_{3}\right): \quad \delta 0.88(\mathrm{t}, J=6.7 \mathrm{~Hz}, 3 \mathrm{H})$, 0.91-1.01 (m, 1H), 1.14-1.43 (m, 21H), 1.64-1.77 (m, 4H), $1.86(\mathrm{~d}, J=12.6 \mathrm{~Hz}, 1 \mathrm{H}), 2.03(\mathrm{dt}, J=$ 7.2, 7.1 Hz, 2H), $2.17(\mathrm{~s}, 1 \mathrm{H}), 3.76(\mathrm{t}, J=6.9 \mathrm{~Hz}, 1 \mathrm{H}), 5.44(\mathrm{dd}, J=15.3,7.5 \mathrm{~Hz}, 1 \mathrm{H}), 5.60(\mathrm{dt}, J=$ 15.3, 7.1 Hz, $1 \mathrm{H}) ;{ }^{13} \mathrm{CNMR}\left(\mathrm{CDCl}_{3}\right): \delta$ 14.1, 22.7, 26.07, 26.15, 26.6, 28.7, 28.8, 29.16, 29.23, 29.3, 29.5, 29.60, 29.61, 31.9, 32.3, 43.7, 77.7, 131.4, 133.1. Anal. Calcd for $\mathrm{C}_{19} \mathrm{H}_{36} \mathrm{O}$ : C, 81.36; H, 12.94. Found: C, 81.20; H, 13.12.

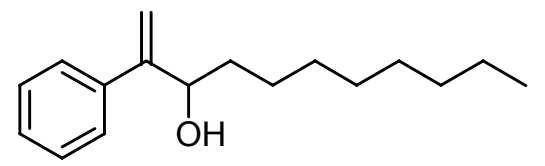

2-Phenyl-1-undecen-3-ol: ${ }^{4}$ IR (neat): 3415, 3080, 3056, 2924, 2854, 1630, 1574, 1494, 1465, 1378, 1131, 1064, 1027, 908, 777, $698 \mathrm{~cm}^{-1} ;{ }^{1} \mathrm{HNMR}\left(\mathrm{CDCl}_{3}\right): \quad \delta 0.87(\mathrm{t}, J=6.9 \mathrm{~Hz}, 3 \mathrm{H})$, 1.19-1.28 (m, 11H), 1.42-1.63 (m, 3H), $1.75(\mathrm{~s}, 1 \mathrm{H}), 4.60-4.64(\mathrm{~m}, 1 \mathrm{H}), 5.30(\mathrm{~s}, 1 \mathrm{H}), 5.35(\mathrm{~s}, 1 \mathrm{H})$, 7.30-7.40 (m, 5H); ${ }^{13} \mathrm{CNMR}\left(\mathrm{CDCl}_{3}\right): \quad \delta 14.1,22.6,25.6,29.2,29.4,29.5,31.8,36.0,73.9,112.5$, $126.9,127.5,128.3,140.1,152.2$. 


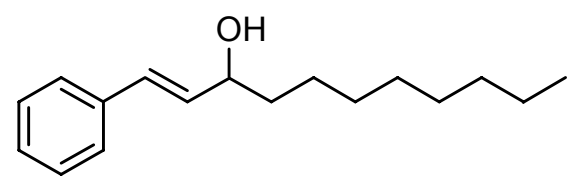

(E)-1-Phenyl-1-undecen-3-ol: 5 IR (neat): 3347, 3103, 3082, 3026, 2926, 2855, 1599, 1494, 1465, 965, 748, $693 \mathrm{~cm}^{-1} ;{ }^{1} \mathrm{HNMR}\left(\mathrm{CDCl}_{3}\right): \quad \delta 0.88(\mathrm{t}, J=6.6 \mathrm{~Hz}, 3 \mathrm{H}), 1.23-1.45(\mathrm{~m}, 12 \mathrm{H})$, $1.55-1.71(\mathrm{~m}, 3 \mathrm{H}), 4.27(\mathrm{dd}, J=6.6,6.3 \mathrm{~Hz}, 1 \mathrm{H}), 6.21(\mathrm{dd}, J=15.9,6.6 \mathrm{~Hz}, 1 \mathrm{H}), 6.56(\mathrm{~d}, J=15.9$ $\mathrm{Hz}, 1 \mathrm{H}), 7.21-7.38(\mathrm{~m}, 5 \mathrm{H}) ;{ }^{13} \mathrm{CNMR}\left(\mathrm{CDCl}_{3}\right): \quad \delta 14.1,22.6,25.4,29.2,29.5,29.6,31.8,37.4$, $73.1,126.4,127.6,128.5,130.1,132.6,136.8$.

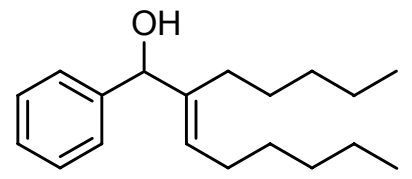

(E)-2-Pentyl-1-phenyl-2-octen-1-ol (6): 6 IR (neat): $\quad 3357,3089,3062,2955,2927,2857$, 1493, 1455, 1378, 1084, 1008, $700 \mathrm{~cm}^{-1} ;{ }^{1} \mathrm{HNMR}\left(\mathrm{CDCl}_{3}\right): \quad \delta 0.83(\mathrm{t}, J=6.9 \mathrm{~Hz}, 3 \mathrm{H}), 0.90(\mathrm{t}, J=$ $6.9 \mathrm{~Hz}, 3 \mathrm{H}), 1.14-1.15(\mathrm{~m}, 12 \mathrm{H}), 1.77$ (d, $J=3.3 \mathrm{~Hz}, 1 \mathrm{H}), 1.77-1.86(\mathrm{~m}, 1 \mathrm{H}), 1.93-2.03(\mathrm{~m}, 1 \mathrm{H})$, $2.07(\mathrm{dt}, J=7.3,7.2 \mathrm{~Hz}, 2 \mathrm{H}), 5.16(\mathrm{~d}, J=2.7 \mathrm{~Hz}, 1 \mathrm{H}), 5.61$ (t, $J=7.2 \mathrm{~Hz}, 1 \mathrm{H}), 7.24-7.37$ (m, 5H); ${ }^{13}{ }^{\mathrm{CNMR}}\left(\mathrm{CDCl}_{3}\right): \quad \delta 14.0,14.1,22.4,22.6,27.6,27.7,29.2,29.5,31.7,32.1,78.2,126.5,127.3$, $127.3,128.2,141.2,142.8$.<smiles>C=C(CCO)C(O)CCc1ccccc1</smiles>

3-Methylene-6-phenyl-hexane-1,4-diol (7): IR (neat): 3353, 3085, 3026, 2927, 2862, 1645, 1603, 1496, 1454, 1320, 1045, 908, 750, $700 \mathrm{~cm}^{-1} ;{ }^{1} \mathrm{HNMR}\left(\mathrm{CDCl}_{3}\right): \delta 1.84-1.98(\mathrm{~m}, 2 \mathrm{H})$, 2.29-2.33 (m, 2H), $2.43(\mathrm{ddd}, J=14.0,8.1,6.0 \mathrm{~Hz}, 1 \mathrm{H}), 2.64(\mathrm{ddd}, J=13.8,9.1,6.8 \mathrm{~Hz}, 1 \mathrm{H}), 2.74$ (ddd, $J=13.9,9.4,6.4 \mathrm{~Hz}, 2 \mathrm{H}), 3.72(\mathrm{ddd}, J=10.4,8.0,4.7 \mathrm{~Hz}, 1 \mathrm{H}), 3.82(\mathrm{dt}, J=10.5,5.4 \mathrm{~Hz}, 1 \mathrm{H})$, $4.13(\mathrm{t}, J=5.4 \mathrm{~Hz}, 1 \mathrm{H}), 4.98(\mathrm{~d}, J=1.2 \mathrm{~Hz}, 1 \mathrm{H}), 5.12(\mathrm{~s}, 1 \mathrm{H}), 7.17-7.31(\mathrm{~m}, 5 \mathrm{H}) ;{ }^{13} \mathrm{CNMR}$ $\left(\mathrm{CDCl}_{3}\right): \quad \delta 32.0,34.8,37.1,62.4,74.9,113.7,125.9,128.41,128.43,141.8,148.8$. Elemental analysis was conducted with its trimethylsilyl ether [bp $60{ }^{\circ} \mathrm{C}$ (bath temp, 1.3 Torr)]. Anal. Calcd for $\mathrm{C}_{19} \mathrm{H}_{34} \mathrm{O}_{2} \mathrm{Si}_{2}$ ： C, 65.08; H, 9.77. Found: C, 65.04; H, 9.85.<smiles>C=C(CCc1ccccc1)C(O)CCCCCCCCC(C)=O</smiles> 
11-Hydroxy-12-(2-phenylethyl)-12-tridecen-2-one: IR (neat): 3428, 3063, 3027, 2929, $2855,1713,1646,1604,1496,1454,1360,1167,900,748,700 \mathrm{~cm}^{-1} ;{ }^{1} \mathrm{HNMR}\left(\mathrm{CDCl}_{3}\right): \quad \delta 1.24-$ $1.28(\mathrm{~m}, 12 \mathrm{H}), 1.51-1.58(\mathrm{~m}, 3 \mathrm{H}), 2.13(\mathrm{~s}, 3 \mathrm{H}), 2.29(\mathrm{dt}, J=15.3,7.9 \mathrm{~Hz} 1 \mathrm{H}), 2.41(\mathrm{t}, J=7.3 \mathrm{~Hz}$, 2H), $2.42(\mathrm{dt}, J=15.3,8.0 \mathrm{~Hz}, 1 \mathrm{H}), 2.80(\mathrm{t}, J=8.2 \mathrm{~Hz}, 2 \mathrm{H}), 4.05-4.09(\mathrm{~m}, 1 \mathrm{H}), 4.92(\mathrm{~d}, J=1.2 \mathrm{~Hz}$, $1 \mathrm{H}), 5.07(\mathrm{~s}, 1 \mathrm{H}), 7.17-7.31(\mathrm{~m}, 5 \mathrm{H}) ;{ }^{13} \mathrm{CNMR}\left(\mathrm{CDCl}_{3}\right): \quad \delta 23.8,25.7,29.1,29.3,29.4,29.5,29.8$, $32.9,34.5,35.5,43,8,75.6,109.9,125.9,128.3,128.3,142.0,151.5,209.3$. Elemental analysis was conducted with its trimethylsilyl ether [bp $155^{\circ} \mathrm{C}$ (bath temp, 0.34 Torr)]. Anal. Calcd for $\mathrm{C}_{24} \mathrm{H}_{40} \mathrm{O}_{2} \mathrm{Si}: \quad \mathrm{C}, 74.17 ; \mathrm{H}, 10.37$. Found: $\mathrm{C}, 74.06 ; \mathrm{H}, 10.56$.

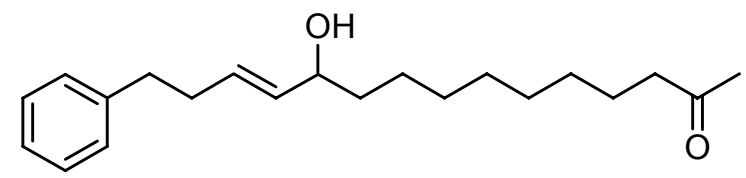

(E)-11-Hydroxy-15-phenyl-12-pentadecen-2-one: IR (neat): 3409, 3026, 2928, 2854, 1715, 1496, 1454, 1361, 969, $747 \mathrm{~cm}^{-1} ;{ }^{1} \mathrm{HNMR}\left(\mathrm{CDCl}_{3}\right): \delta 1.20-1.31(\mathrm{~m}, 12 \mathrm{H}), 1.43-1.58(\mathrm{~m}$, $3 \mathrm{H}), 2.13(\mathrm{~s}, 3 \mathrm{H}), 2.36$ (dt, $J=7.7,7.8 \mathrm{~Hz}, 2 \mathrm{H}), 2.41$ (t, $J=7.5 \mathrm{~Hz}, 2 \mathrm{H}), 2.70(\mathrm{dd}, J=8.1,7.2 \mathrm{~Hz}$, 2H), $4.01(\mathrm{dt}, J=6.6,6.6 \mathrm{~Hz}, 1 \mathrm{H}), 5.46(\mathrm{dd}, J=15.3,6.9 \mathrm{~Hz}, 1 \mathrm{H}), 5.66(\mathrm{dt}, J=15.3,6.6 \mathrm{~Hz}, 1 \mathrm{H})$,

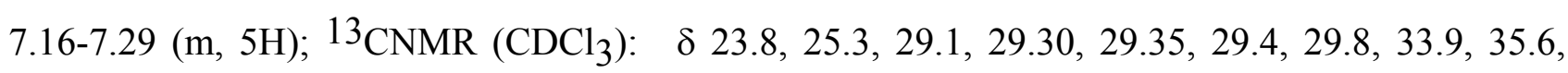
$37.2,43.8,73.0,125.8,128.3,128.4,130.9,133.9,141.7,209.3$. Elemental analysis was conducted with its trimethylsilyl ether [bp $150{ }^{\circ} \mathrm{C}$ (bath temp, 0.3 Torr)]. Anal. Calcd for $\mathrm{C}_{24} \mathrm{H}_{40} \mathrm{O}_{2} \mathrm{Si}: \quad \mathrm{C}, 74.17 ; \mathrm{H}, 10.37$. Found: $\mathrm{C}, 74.29 ; \mathrm{H}, 10.42$.

\section{References}

1. Inomata, K.; Igarashi, S.; Mohri, M.; Yamamoto, T.; Kinoshita, H.; Kotake, H. Chem. Lett. $1987,707$.

2. Takai, K.; Tagashira, M.; Kuroda, T.; Oshima, K.; Utimoto, K.; Nozaki, H. J. Am. Chem. Soc. 1986, $108,6048$.

3. Kitagawa, K.; Inoue, A.; Shinokubo, H.; Oshima, K. Angew. Chem. Int. Ed. 2000, 39, 2481.

4. Takai, K.; Kimura, K.; Kuroda, T.; Hiyama, T.; Nozaki, H. Tetrahedron Lett. 1983, 24, 5281.

5. Vettel, S.; Vaupel, A.; Knochel, P. J. Org. Chem. 1996, 61, 7473.

6. Takai, K.; Kataoka, Y.; Utimoto, K. J. Org. Chem. 1990, 55, 1707; Inoue, A.; Kitagawa, K.; Shinokubo, H.; Oshima, K. J. Org. Chem. 2001, 66, 4333. 